\title{
8 REGULAÇÃO EMOCIONAL E QUALIDADE DO RELACIONAMENTO COM OS PAIS COMO PREDITORAS DE SINTOMATOLOGIA DEPRESSIVA EM ADOLESCENTES ${ }^{1}$
}

\author{
| Joana Antunes²; Ana Paula Matos ${ }^{3}$; José Joaquim Costa $^{4} \mid$
}

\section{RESUMO}

CONTEXTO: A adolescência é um período de risco para o desenvolvimento da sintomatologia depressiva, com a regulação emocional e a qualidade do relacionamento com os pais a desempenharem um papel importante no desenvolvimento da Depressão.

OBJETIVO(S): Analisar longitudinalmente os efeitos preditores das estratégias de regulação emocional e da qualidade do relacionamento com os pais na sintomatologia depressiva.

METODOLOGIA Um total de 566 adolescentes com 13-17 anos de idade preencheram questionários em dois momentos de avaliação, com um intervalo de 6 meses.

RESULTADOS: Após o controlo dos efeitos do género, as estratégias de regulação emocional e a qualidade do relacionamento com os pais (T1) explicaram 36\% e 21,9\% da variância da sintomatologia depressiva (T2), respetivamente. A autocrítica, a catastrofização e a ruminação foram preditoras positivas e significativas, e a reavaliação positiva e o replaneamento foram preditoras negativas significativas. Uma maior perceção de conflito nos relacionamentos com os pais e uma menor perceção de suporte no relacionamento com a mãe previu níveis mais elevados de sintomatologia depressiva.

CONCLUSÕES: Os resultados apresentam implicações na prevenção e no tratamento da Depressão na adolescência, tais como abordar o uso de estratégias maladaptativas de regulação emocional e a existência de conflito no relacionamento com os pais, e aumentar os efeitos protetores associados às estratégias adaptativas de regulação emocional e à perceção de qualidades positivas nos relacionamentos.

PALAVRAS-CHAVE: Depressão; Emoções; Relações familiares; Adolescente

\section{RESUMEN}

"Regulación emocional y calidad de la relación con padres como predictores de sintomatología depresiva en adolescentes ${ }^{1 "}$

CONTEXTO: La adolescencia es un período de riesgo para el desarrollo de la sintomatología depresiva, con la regulación emocional y la calidad de la relación con los padres a desempeñar un importante papel en el desarrollo de la Depresión.

OBJETIVO(S): Analizar longitudinalmente los efectos predictores de las estrategias de regulación emocional y de la calidad de la relación con los padres en la sintomatología depresiva.

METODOLOGÍA: Un total de 566 adolescentes con 13-17 años de edad llenaron cuestionarios en dos momentos de evaluación, con un intervalo de 6 meses. RESULTADOS: Después del control de los efectos del género, las estrategias de regulación emocional y la calidad de la relación con los padres (T1) explicaron el $36 \%$ y el $21,9 \%$ de la varianza de la sintomatología depresiva (T2), respectivamente. La autocrítica, la catastrofización y la rumiación fueron predictoras positivas y significativas, y la reevaluación positiva y el replanteamiento fueron predictores negativos significativos. Una mayor percepción de conflicto en las relaciones con los padres y una menor percepción de soporte en la relación con la madre predijo niveles más elevados de sintomatología depresiva.

CONCLUSIONES: Los resultados presentan implicaciones en la prevención y el tratamiento de la depresión en la adolescencia, tales como abordar el uso de estrategias maladaptativas de regulación emocional y la existencia de conflicto en la relación con los padres, y aumentar los efectos protectores asociados a las estrategias adaptativas de regulación emocional y a la percepción de cualidades positivas en las relaciones.

\section{DESCRIPTORES: Depresión; Emociones; Relaciones familiares; Adolescente}

\begin{abstract}
"Emotional regulation and quality of the relationship with parents as predictors of depressive symptomatology in adolescents"

BACKGROUND: Adolescence is a time of risk for the development of depressive symptomatology, with emotional regulation and the quality of the relationship with the parents playing an important role in the development of Depression.

AIM: To analyze longitudinally the predictive effects of the emotional regulation strategies and the quality of the relationship with the parents in the depressive symptomatology.

METHODS: A total of 566 adolescents with 13-17 years old completed questionnaires at two moments of evaluation, with a 6-month interval.

RESULTS: After controlling gender effects, emotional regulation strategies and the quality of the relationship with the parents (T1) explained $36 \%$ and $21.9 \%$ of the variance of the depressive symptomatology (T2), respectively. Self-blame, catastrophizing, and rumination were positive significant predictors, and positive reappraisal and refocus on planning were negative significant predictors. A greater perception of conflict in the relationships with the parents and a lower perception of support in the relationship with the mother predicted higher levels of depressive symptomatology. CONCLUSIONS: The results have implications for the prevention and treatment of depression in adolescence, such as addressing the use of maladaptive emotional regulation strategies and the existence of conflict in the relationship with parents, and increasing the protective effects associated with adaptive emotional regulation strategies and the perception of positive qualities in the relationships.
\end{abstract}

\section{KEYWORDS: Depression; Emotions; Family relations; Adoles-} cent

Submetido em 30-12-2017

Aceite em 30-03-2018

1 Este artigo foi extraído da Dissertação de Mestrado da primeira autora, sobre o tema: "Estudo longitudinal do efeito moderador da qualidade do relacionamento com os pais na relação entre regulação emocional e sintomatologia depressiva nos adolescentes” (2017) - apresentada na Faculdade de Psicologia e Ciências da Educação da Universidade de Coimbra.

2 Mestre em Psicologia, joana.simoes.antunes@gmail.com

3 Doutorada em Psicologia; Investigadora do Centro de Investigação do Núcleo de Estudos e Intervenção Cognitivo-Comportamental; Professora Associada na Faculdade de Psicologia e Ciências da Educação da Universidade de Coimbra, Rua do Colégio Novo, 3000-115, Coimbra, Portugal, apmatos@fpce.uc.pt

4 Doutorado em Psicologia; Investigador no Centro de Investigação do Núcleo de Estudos e Intervenção Cognitivo-Comportamental; Professor Auxiliar na Faculdade de Psicologia e Ciências da Educação da Universidade de Coimbra, 3000-115, Coimbra, Portugal, jjcosta@fpce.uc.pt

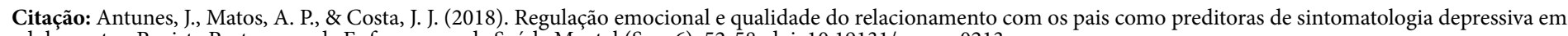
adolescentes. Revista Portuguesa de Enfermagem de Saúde Mental (Spe. 6), 52-58. doi: 10.19131/rpesm.0213 


\section{INTRODUÇÃO}

A depressão tem sido apontada como uma das maiores causas de incapacidade no mundo, com a adolescência a revelar-se como um período de elevadas taxas de prevalência de sintomatologia depressiva, principalmente nas raparigas (Erse et al., 2016; Brito, Matos, Pinheiro e Monteiro, 2015; Ferreira, 2016). Quando o início da depressão ocorre na adolescência, a perturbação apresenta um curso relativamente estável e mais severo ao longo do tempo, com um maior risco de recorrência (Arnarson et al., 2015).

A depressão envolve dificuldades ao nível da regulação emocional, com os indivíduos a revelarem dificuldades em experienciar as emoções (Arnarson et al., 2015; Duarte, 2014; Ferreira, 2016; Garnefski, Boon \& Kraaij, 2003; Garnefski \& Kraaij, 2006; Stikkelbroek, Bodden, Kleinjan, Reijnders \& Baar, 2016). É importante notar que só na fase final da adolescência é que se verifica uma aquisição completa das competências associadas à regulação emocional, o que pode levar a dificuldades na regulação cognitiva das emoções e a maior vulnerabilidade ao desenvolvimento de psicopatologia perante acontecimentos de vida negativos (Garnefski et al., 2003), principalmente ao considerar que as exigências desenvolvimentais (e.g. procura de maior autonomia, aumento do conflito com os pais e o desafio emocional de experiências românticas e sexuais) podem exceder as capacidades regulatórias dos mais vulneráveis (Yap, Allen \& Sheeber, 2007).

Entre as tarefas desenvolvimentais associadas à adolescência encontra-se a individualização, que se caracteriza pela diminuição da dependência em relação a pessoas significativas e o aumento da autonomia (Levpušček, 2006). No entanto, os pais permanecem como figuras de vinculação importantes, sendo que a satisfação de necessidades relacionais na família, combinada com uma autonomia não excessiva, encontra-se associada a melhor ajustamento psicossocial (Levpušček, 2006). Estas relações de vinculação são influenciadas pela qualidade do funcionamento familiar que inclui fatores como a disponibilidade de suporte, coesão, comunicação e níveis de conflito (Rawatlal, Kliewer \& Pillay, 2015).

No âmbito da revisão de literatura, o presente estudo teve como objetivo estudar os efeitos preditores das estratégias de regulação emocional e da qualidade do relacionamento com os pais no desenvolvimento de sintomatologia depressiva em adolescentes portugueses.

\section{METODOLOGIA}

O presente estudo longitudinal foi autorizado pela Comissão Nacional de Proteção de Dados (CNPD) e pela Direção Geral de Inovação e Desenvolvimento Curricular (DGIDC), inserindo-se no projeto de investigação "Prevenção da Depressão em Adolescentes Portugueses: Estudo de eficácia de uma intervenção com adolescentes e pais" (PTDC/MHCPCL/4824/2012), financiado pela Fundação para a Ciência e Tecnologia (FCT) e pela Realan Foundation. A amostra comunitária foi constituída por 566 adolescentes (60.25\% do sexo feminino) com idades entre os 13 e os 17 anos $(\mathrm{M}=14.43$; $\mathrm{DP}=867)$, distribuindose pelos níveis socioeconómicos baixo (48.4\%), médio (36.6\%) e elevado (15\%).

Não se verificaram diferenças estatisticamente significativas entre os géneros em termos da idade $[t$ $(429.398)=.404, \mathrm{p}=.686]$ e do nível socioeconómico $\left[\mathrm{X}^{2}(2)=.195, \mathrm{p}=.907\right]$.

A amostra foi recolhida em escolas públicas e privadas da região centro de Portugal através do preenchimento de questionários em dois momentos de avaliação, com um intervalo de seis meses. Foi assegurada a confidencialidade a todos os estudantes que participaram voluntariamente, tendo sido requerido o consentimento informado do estudante e do seu responsável legal.

No primeiro momento de avaliação (T1) foi recolhida informação sociodemográfica e foi aplicado o Inventory of the Quality of Interpersonal Relations IQRI (versão portuguesa para adolescentes: Marques, Matos e Pinheiro, 2014; Marques, Pinheiro, Matos e Marques, 2015) e o Cognitive Emotion Regulation Questionnaire - CERQ (versão portuguesa: Matos, Cherpe e Serra, 2012).

O IQRI analisa a perceção do adolescente em relação à qualidade do seu relacionamento com a mãe e com o pai ao nível de três dimensões: suporte (disponibilidade de ajuda perante determinadas situações); profundidade (estima e comprometimento com a relação); e conflito (experienciar sentimentos negativos ou ambivalentes na relação).

O CERQ avalia as estratégias cognitivas de regulação emocional usadas perante um acontecimento de vida negativo ou traumático, sendo composto por nove subescalas: autocrítica; ruminação; catastrofização; culpar o outro; aceitação; reavaliação positiva; replaneamento; reorganização positiva; e reperspetivar. 
No segundo momento de avaliação (T2) foi aplicado o Children Depression Inventory - CDI (versão portuguesa: Marujo, 1994), que permite avaliar a sintomatologia depressiva em crianças e adolescentes. No presente estudo recorreu-se ao valor da escala total.

O tratamento estatístico dos dados foi efetuado através do programa Statistical Package for Social Sciences (SPSS), versão 22.0.0.2 para Windows.

\section{RESULTADOS}

\section{Estatísticas Descritivas e Diferenças de Acordo com o Género}

De forma a verificar a necessidade de controlar o efeito do género, foi analisada a possível existência de diferenças recorrendo a testes t-Student para amostras independentes e calculado o Eta Quadrado para examinar a magnitude do efeito. Os resultados (Quadro 1) revelaram diferenças estatisticamente significativas, mas com uma magnitude de efeito baixa. As raparigas apresentaram níveis superiores de sintomatologia depressiva, recorreram mais à autocrítica, ruminação e aceitação, e apresentaram níveis superiores de suporte, profundidade e conflito no relacionamento com a mãe, e níveis superiores de profundidade no relacionamento com o pai. Os rapazes mostraram recorrer mais do que as raparigas à estratégia de regulação emocional de culpar o outro.

Quadro 1 - Consistência interna ( $\alpha$ ), estatísticas descritivas (M e DP), e análise das diferenças de acordo com o género ( $t$ de Student) e da magnitude do efeito $\left(\eta^{2}\right)$

\begin{tabular}{|c|c|c|c|c|c|c|c|c|c|c|}
\hline \multirow[b]{2}{*}{ Medidas } & \multirow[b]{2}{*}{$a$} & \multicolumn{2}{|c|}{$\begin{array}{l}\text { Amostra total } \\
(\mathrm{n}=566)\end{array}$} & \multicolumn{2}{|c|}{$\begin{array}{c}\text { Grupo Masculino } \\
(\mathrm{n}=225)\end{array}$} & \multicolumn{2}{|c|}{$\begin{array}{c}\text { Grupo Feminino } \\
(\mathrm{n}=341)\end{array}$} & \multirow[b]{2}{*}{$\mathrm{t}$} & \multirow[b]{2}{*}{$\mathrm{p}$} & \multirow[b]{2}{*}{$\eta^{2}$} \\
\hline & & M & DP & M & DP & M & DP & & & \\
\hline CDI (T2) & .905 & 9.747 & 7.353 & 7.877 & 6.131 & 10.981 & 7.826 & -5.272 & .000 & .047 \\
\hline \multicolumn{11}{|c|}{ CERQ (T1) } \\
\hline F1 & .776 & 9.140 & 3.212 & 8.609 & 2.889 & 9.490 & 3.367 & -3.324 & .001 & .019 \\
\hline F2 & .819 & 10.734 & 3.744 & 9.759 & 3.338 & 11.377 & 3.862 & -5.296 & .000 & .047 \\
\hline F3 & .815 & 8.406 & 3.567 & 8.237 & 3.324 & 8.517 & 3.719 & -.914 & .361 & .001 \\
\hline F4 & .737 & 7.058 & 2.549 & 7.360 & 2.498 & 6.858 & 2.566 & 2.300 & .022 & .009 \\
\hline F5 & .706 & 11.423 & 3.430 & 10.982 & 3.224 & 11.713 & 3.534 & -2.543 & .011 & .011 \\
\hline F6 & .849 & 11.494 & 4.032 & 11.679 & 3.747 & 11.372 & 4.210 & .910 & .363 & .001 \\
\hline F7 & .791 & 12.489 & 3.590 & 12.438 & 3.498 & 12.523 & 3.654 & -.278 & .781 & .000 \\
\hline F8 & .825 & 12.327 & 3.802 & 12.284 & 3.472 & 12.356 & 4.009 & -.227 & .820 & .000 \\
\hline F9 & .785 & 10.971 & 3.704 & 10.655 & 3.621 & 11.180 & 3.748 & -1.653 & .099 & .005 \\
\hline \multicolumn{11}{|c|}{ IQRI - Mãe (T1) } \\
\hline F1 & .862 & 3.257 & .720 & 3.140 & .747 & 3.334 & .691 & -3.163 & .002 & .017 \\
\hline F2 & .867 & 2.069 & .688 & 1.991 & .656 & 2.120 & .705 & -2.182 & .030 & .008 \\
\hline F3 & .876 & 3.424 & .611 & 3.286 & .677 & 3.514 & .545 & -4.217 & .000 & .031 \\
\hline \multicolumn{11}{|c|}{ IQRI - Pai (T1) } \\
\hline F1 & .907 & 3.000 & .795 & 3.001 & .782 & 3.000 & .804 & .009 & .992 & .000 \\
\hline F2 & .873 & 2.041 & .668 & 2.051 & .656 & 2.035 & .676 & .277 & .782 & .000 \\
\hline F3 & .911 & 3.243 & .747 & 3.163 & .723 & 3.297 & .758 & -2.069 & .039 & .008 \\
\hline
\end{tabular}

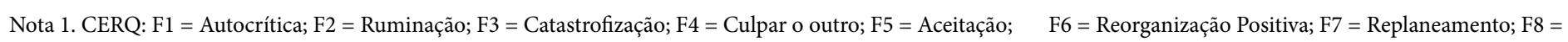
Reavaliação Positiva; F9 = Reperspetivar

Nota 2. IQRI: F1 = Suporte; F2 = Conflito; F3 = Profundidade

\section{Estratégias de Regulação Emocional como Preditoras da Sintomatologia Depressiva}

Foram realizadas análises preliminares que garantiram a adequação dos dados a análises de Regressão Múltipla Hierárquica (Quadro 2). 
Quadro 2 - Análise de Regressão Múltipla com as estratégias cognitivas de regulação emocional (CERQ) como preditoras de sintomatologia depressiva (CDI) para amostra total (controlando o género)

\begin{tabular}{|l|l|l|l|l|l|l|}
\hline $\begin{array}{l}\text { Mod- } \\
\text { elo }\end{array}$ & Preditores & $\mathrm{R}^{2}$ & $\mathrm{~F}$ & $\beta$ & $\mathrm{t}$ & $\mathrm{p}$ \\
\hline 1 & Género & .043 & $25.192^{* * *}$ & .207 & 5.019 & .000 \\
\hline 2 & Género & .403 & $37.426^{* * *}$ & .132 & 3.829 & .000 \\
\hline & $\begin{array}{l}\text { CERQ (di- } \\
\text { mensões) }\end{array}$ & & & & & \\
\cline { 2 - 7 } & Autocrítica & & & .382 & 8.183 & .000 \\
\hline & Ruminação & & & .128 & 2.440 & .015 \\
\hline & $\begin{array}{l}\text { Catastrofiza- } \\
\text { ção }\end{array}$ & & & .131 & 2.671 & .008 \\
\cline { 2 - 7 } & $\begin{array}{l}\text { Culpar o } \\
\text { outro }\end{array}$ & & & .043 & 1.128 & .260 \\
\cline { 2 - 7 } & Aceitação & & & -.035 & -.751 & .453 \\
\hline & $\begin{array}{l}\text { Reorganiza- } \\
\text { ção positiva }\end{array}$ & & & .032 & .666 & .506 \\
\cline { 2 - 7 } & $\begin{array}{l}\text { Replanea- } \\
\text { mento }\end{array}$ & & & -.138 & -2.589 & .010 \\
\cline { 2 - 7 } & $\begin{array}{l}\text { Reavaliação } \\
\text { positiva }\end{array}$ & & & -.208 & -3.696 & .000 \\
\cline { 2 - 7 } & $\begin{array}{l}\text { Reperspe- } \\
\text { tivar }\end{array}$ & & & .059 & 1.273 & .203 \\
\hline
\end{tabular}

Inseriu-se a variável género em primeiro lugar, tendo sido gerado um modelo estatisticamente significativo, em que este explicou $4.3 \%$ da variância de sintomatologia depressiva.

O segundo modelo, onde constam as estratégias de regulação emocional (T1), foi estatisticamente significativo, explicando $36 \%$ da variância da sintomatologia depressiva em T2 $\left[\Delta \mathrm{R}^{2}=.360 ; \mathrm{F}(9,555)=37.170\right.$, $\mathrm{p}$ $<.001]$. As estratégias de regulação emocional de autocrítica, catastrofização e ruminação foram preditoras positivas e significativas de sintomatologia depressiva no segundo momento de avaliação. A reavaliação positiva e o replaneamento apresentaram-se como preditoras negativas e significativas de sintomatologia depressiva.

\section{Qualidade do Relacionamento com os Pais como Preditora da Sintomatologia Depressiva}

Análises preliminares garantiram a adequação dos dados a análises de Regressão Múltipla Hierárquica (Quadro 3).
Quadro 3 - Análise de Regressão Múltipla com a qualidade do relacionamento com os pais (IQRI) como preditora de sintomatologia depressiva (CDI) para amostra total (controlando o género)

\begin{tabular}{|l|l|l|l|l|l|l|}
\hline $\begin{array}{l}\text { Mod- } \\
\text { elo }\end{array}$ & Preditores & $\mathrm{R}^{2}$ & $\mathrm{~F}$ & $\beta$ & $\mathrm{t}$ & $\mathrm{p}$ \\
\hline 1 & Género & .043 & $24.388^{* * *}$ & .207 & 4.938 & .000 \\
\hline 2 & Género & .261 & $27.300^{* * *}$ & .215 & 5.580 & .000 \\
\hline & $\begin{array}{l}\text { IQRI (di- } \\
\text { mensões) }\end{array}$ & & & & & \\
\cline { 2 - 7 } & $\begin{array}{l}\text { Suporte - } \\
\text { mãe }\end{array}$ & & & -.226 & -3.575 & .000 \\
\cline { 2 - 7 } & $\begin{array}{l}\text { Conflito - } \\
\text { mãe }\end{array}$ & & & .158 & 2.951 & .003 \\
\cline { 2 - 7 } & $\begin{array}{l}\text { Profundi- } \\
\text { dade - mãe }\end{array}$ & & & -.005 & -.087 & .931 \\
\cline { 2 - 7 } & Suporte - pai & & & -.116 & -1.687 & .092 \\
\cline { 2 - 7 } & $\begin{array}{l}\text { Conflito - } \\
\text { pai }\end{array}$ & & & .197 & 3.835 & .000 \\
\cline { 2 - 7 } & $\begin{array}{l}\text { Profundi- } \\
\text { dade - pai }\end{array}$ & & & .117 & 1.736 & .083 \\
\hline
\end{tabular}

${ }^{* * *} \mathrm{p} \leq 0.001$

Para controlar o seu efeito, a variável género foi inserida em primeiro lugar, tendo sido gerado um modelo estatisticamente significativo, com este a explicar $4.3 \%$ da variância de sintomatologia depressiva.

O segundo modelo gerado, constituído pelas dimensões do IQRI (T1), revelou-se estatisticamente significativo, explicando $21.9 \%$ da variância da sintomatologia depressiva em $\mathrm{T} 2\left[\Delta \mathrm{R}^{2}=.219 ; \mathrm{F}(6,540)=26.640\right.$, $\mathrm{p}<.001]$. Os resultados obtidos sugerem que uma maior perceção de conflito nos relacionamentos com os pais e uma menor perceção de suporte no relacionamento com a mãe, no primeiro momento de avaliação, predizem níveis mais elevados de sintomatologia depressiva no segundo momento de avaliação.

\section{DISCUSSÃO}

A literatura sugere que a regulação emocional (Arnarson et al., 2015; Duarte, 2014; Ferreira, 2016; Garnefski et al., 2003; Garnefski \& Kraaij, 2006; Stikkelbroek et al., 2016) e a qualidade do relacionamento com os pais (Brito, Matos, Pinheiro e Monteiro, 2015; Sheeber, Davis, Leve, Hops \& Tildesley, 2007; Yap, Pilkington, Ryan \& Jorm, 2014) podem desempenhar um papel importante no desenvolvimento da depressão, pelo que o presente estudo longitudinal teve como principal objetivo examinar os efeitos preditores destas variáveis na sintomatologia depressiva em adolescentes portugueses. 
No que diz respeito aos efeitos preditores da regulação emocional, após se controlar os efeitos do género, o modelo em que se encontram as estratégias de regulação emocional apresentou-se como estatisticamente significativo, com as estratégias avaliadas em T1 a explicarem $36 \%$ da variância da sintomatologia depressiva em T2. Estes resultados vão ao encontro do que foi observado por Garnefski \& Kraaij (2006) num estudo transversal, em que as estratégias de regulação emocional avaliadas pelo CERQ explicaram 37.9\% e $32.1 \%$ da variância da sintomatologia depressiva no início (12-15 anos) e no final (16-18 anos) da adolescência, respetivamente.

As estratégias de autocrítica, catastrofização e ruminação foram preditoras positivas e significativas de sintomatologia depressiva em T2, revelando-se como estratégias maladaptativas de regulação emocional. $\mathrm{O}$ mesmo se verificou noutros estudos transversais ( $\mathrm{Du}$ arte, 2014; Garnefski et al., 2003; Garnefski \& Kraaij, 2006; Stikkelbroek et al., 2016) e longitudinais (Arnarson et al., 2015; Ferreira, 2016).

As estratégias de reavaliação positiva e replaneamento foram preditoras negativas e significativas de sintomatologia depressiva em T2. Outros estudos também apontaram como estratégias adaptativas a reavaliação positiva (Ferreira, 2016; Garnefski et al., 2003; Garnefski \& Kraaij, 2006; Stikkelbroek et al., 2016) e o replaneamento (Duarte, 2014; Ferreira, 2016; Stikkelbroek et al., 2016).

O modelo gerado com as dimensões do IQRI apresentou-se estatisticamente significativo, com as várias dimensões avaliadas em T1 a explicarem $21.9 \%$ da variância da sintomatologia depressiva em T2. Tal vai ao encontro dos resultados do estudo transversal realizado por Brito, Matos, Pinheiro e Monteiro (2015), em que a qualidade do relacionamento com os pais avaliada pelo IQRI explicou $17.1 \%$ da variância da sintomatologia depressiva nos adolescentes.

Os resultados obtidos sugerem que uma maior perceção de conflito nos relacionamentos com os pais em T1 prediz níveis mais elevados de sintomatologia depressiva em T2. Apesar de ser considerado normativo na adolescência a existência de algum grau de conflito no relacionamento com os pais, situações em que os níveis de conflito são prolongados, intensos e repetidos têm sido associadas a dificuldades no ajustamento psicológico e a maior sintomatologia depressiva (Sheeber et al., 2007; Yap et al., 2014).
Os resultados sugeriram igualmente que uma menor perceção de suporte no relacionamento com a mãe em T1 prediz níveis mais elevados de sintomatologia depressiva em T2, corroborando os resultados de outros estudos (Sheeber et al., 2007; Yap et al., 2014). Contudo, no presente estudo, o suporte no relacionamento com o pai não se revelou como um preditor significativo de sintomatologia depressiva, o que poderá ser explicado por a mãe poder exercer um maior impacto enquanto fonte de suporte na presente amostra ou por existirem possíveis diferenças de acordo com o género. A este respeito, é importante notar que no estudo longitudinal realizado por Branje, Hale, Frijns \& Meeus (2010), a qualidade do relacionamento com a mãe relacionou-se com a sintomatologia depressiva apresentada pelas raparigas e pelos rapazes um ou dois anos depois, enquanto no relacionamento com o pai, esta relação só se verificou nos rapazes.

\section{CONCLUSÃO}

Os resultados encontrados no presente estudo possibilitam uma melhor compreensão sobre os fatores de proteção e de risco no desenvolvimento de sintomatologia depressiva. É importante notar que um relacionamento positivo entre pais e filhos tem sido associado a vários indicadores de desenvolvimento positivos que funcionam como fatores de proteção do desenvolvimento de sintomatologia depressiva (Branje, Hale, Frijns \& Meeus, 2010; Ewing, Diamond \& Levy, 2015). Segundo o modelo transacional, fatores ambientais interagem com fatores individuais, influenciando uma trajetória mais ou menos adaptativa e o possível desenvolvimento de sintomatologia depressiva (Ewing et al., 2015). Os resultados do presente estudo apontam para a importância de investigarmos, no futuro, as relações que se estabelecem entre a regulação emocional e a qualidade do relacionamento com os pais na predição da depressão, até porque são escassos os dados sobre a influência da interação entre o contexto e a regulação emocional no desenvolvimento de sintomatologia depressiva (Naragon-Gainey, McMahon \& Chacko, 2017). Apesar da relevância dos resultados encontrados, existem algumas fragilidades no presente estudo. Os dados apresentados foram recolhidos a partir de uma amostra comunitária, pelo que seria do nosso interesse replicar este estudo em amostra clínica. É igualmente importante notar que não foram analisadas as possíveis diferenças de acordo com a idade nas variáveis de estudo, sendo pertinente estudar no futuro o contributo destas diferenças no desenvolvimento da sintomatologia depressiva. 


\section{IMPLICAÇÕES PARA A PRÁTICA CLÍNICA}

Ao possibilitar uma melhor compreensão sobre os fatores de proteção e de risco no desenvolvimento de sintomatologia depressiva, o presente estudo permitiu igualmente um melhor conhecimento sobre os possíveis alvos de intervenção no tratamento e na prevenção da depressão na adolescência.

Os resultados evidenciam o recurso a estratégias de regulação emocional maladaptativas como um fator de risco para o desenvolvimento de sintomatologia depressiva na adolescência. De acordo com Garnefski, Boon \& Kraaij (2003), a associação entre estratégias maladaptativas e sintomatologia depressiva na adolescência ocorre independentemente do tipo de acontecimento de vida que leva ao recurso a estas estratégias. Desta forma, trabalhar aspetos como a psicoeducação sobre estratégias de regulação emocional e incentivar o uso adequado de estratégias adaptativas revela-se fundamental, permitindo a prevenção de um estilo de coping maladaptativo e a promoção do recurso a estratégias adaptativas que podem ser implementadas em vários tipos de acontecimentos negativos.

Para além da importância de aumentar a perceção de qualidades positivas e incentivar a existência de suporte e envolvimento por parte dos pais nos relacionamentos com os seus filhos, especialmente o suporte por parte da mãe, os resultados obtidos dão igualmente uma maior relevância a trabalhar questões associadas ao conflito no relacionamento entre pais e filhos.

\section{REFERÊNCIAS BIBLIOGRÁFICAS}

Arnarson, E. Ö, Matos, A. P., Salvador, C., Ribeiro, C., Sousa, B., \& Craighead, W. E. (2015). Longitudinal Study of Life Events, Well-Being, Emotional Regulation and Depressive Symptomatology. Journal of Psychopathology and Behavioral Assessment, 38 (2), 159171. Doi: 10.1007/s10862-015-9524-8.

Branje, S. J. T., Hale, W. W., Frijns, T., \& Meeus, W. H. J. (2010). Longitudinal Associations Between Perceived Parent-Child Relationship Quality and Depressive Symptoms in Adolescence. Journal of Abnormal Child Psychology, 38 (6), 751-763. Doi: 10.1007/ s10802-010-9401-6
Brito, J., Matos, A. P., Pinheiro, M. R., e Monteiro, S. (2015). Quality of Interpersonal Relationships and Depression in Adolescence: Psychosocial Functioning Moderating Effect. The European Proceedings of Social \& Behavioural Sciences, 81-94. Doi: 10.15405/ epsbs.2015.08.8

Duarte, A. C. M. (2014). O efeito moderador do género na relação entre as estratégias cognitivas de regulação emocional e a sintomatologia depressiva, numa amostra de adolescentes portugueses. (Dissertação de Mestrado não publicada). Faculdade de Psicologia e de Ciências da Educação da Universidade de Coimbra, Portugal.

Erse, M. P. Q. A., Simões, R. M. P., Façanha, J. D. N., Marques, L. A. F. A. M., Loureiro, C. R. E. C., Matos, M. E. T. S., \& Santos, J. C. P. (2016). Adolescent depression in schools: + Contigo Project. Revista de Enfermagem Referência, 4 (9), 37-44. Doi: 10.12707/ RIV15026.

Ewing, E. S. K., Diamond, G., \& Levy, S. (2015). Attachment-based family therapy for depressed and suicidal adolescents: theory, clinical model and empirical support. Attachment \& Human Development, 17 (2), 136-156. Doi: 10.1080/14616734.2015.1006384.

Ferreira, N. A. (2016). Emotion regulation as a predictor of depression and psychosocial impairment in adolescence moderated by negative life events. (Dissertação de Mestrado não publicada). Faculdade de Psicologia e de Ciências da Educação da Universidade de Coimbra, Portugal.

Garnefski, N., Boon, S., \& Kraaij, V (2003). Relationships Between Cognitive Strategies of Adolescents and Depressive Symptomatology Across Different Types of Life Event. Journal of Youth and Adolescence, 32 (6), 401-408. Doi: 10.1023/A:1025994200559.

Garnefski, N., Kraaij, V. (2006). Relationships between cognitive emotion regulation strategies and depressive symptoms: A comparative study of five specific samples. Personality and Individual Differences, 40 (8), 1659-1669. Doi: 10.1016/j.paid.2005.12.009. 
Levpušček, M. P. (2006). Adolescent individuation in relation to parents and friends: Age and gender differences. European Journal of Developmental Psychology, 3 (3), 238-264. Doi: 10.1080/17405620500463864.

Marques, D., Matos, A. P., e Pinheiro, M. R. (2014). Estudo da estrutura fatorial da versão mãe do IQRI para adolescentes. Psicologia, Saúde \& Doenças, 15 (1), 234-244. Doi: 10.15309/14psd150119.

Marques, D., Pinheiro, M. R., Matos, A. P., e Marques, C. (2015). Confirmatory Factor Analysis of the QRI Father's Version in a Portuguese sample of adolescents. Procedia-Social and Behavioral Sciences, 165, 267-274. Doi: 10.1016/j.sbspro.2014.12.631.

Marujo, H. (1994). Síndromas depressivos na infância e na adolescência. (Tese de Doutoramento não publicada). Faculdade de Psicologia e de Ciências da Educação da Universidade de Lisboa, Portugal.

Matos, A. P., Cherpe, S., e Serra, A. R. (2012). Estudo psicométrico do Questionário de Regulação Cognitiva da Emoção (CERQ), em adolescentes Portugueses (manuscrito não publicado).

Naragon-Gainey, K., McMahon, T. P., \& Chacko, T. P. (2017). The Structure of Common Emotion Regulation Strategies: A Meta-Analytic Examination. Psychological Bulletin, 143 (4), 384-427. Doi: 10.1037/bul0000093.
Rawatlal, N., Kliewer, W., \& Pillay, B. J. (2015). Adolescent attachment, family functioning and depressive symptoms. South African Journal of Psychiatry, 21 (3), 80-85. Doi: 10.7196/SAJP.8252.

Sheeber, L. B., Davis, B., Leve, C., Hops, H., \& Tildesley, E. (2007). Adolescents' Relationships with Their Mothers and Fathers: Associations with Depressive Disorder and Subdiagnostic Symptomatology. Journal of Abnormal Psychology, 116 (1), 144-154. Doi: 10.1037/0021-843X.116.1.144.

Stikkelbroek, Y., Bodden, D. H. M., Kleinjan, M., Reijnders, M., \& Baar, A. L. (2016). Adolescent Depression and Negative Life Events, the Mediating Role of Cognitive Emotion Regulation. PloS one, 11 (8), 1-16. Doi: 10.1371/journal.pone.0161062.

Yap, M. B. H., Allen, N. B., \& Sheeber, L. (2007). Using an Emotion Regulation Framework to Understand the Role of Temperament and Family Processes in Risk for Adolescent Depressive Disorders. Clinical Child and Family Psychology, 10 (2), 180-196. Doi: 10.1007/ s10567-006-0014-0.

Yap, M. B. H., Pilkington, P. D., Ryan, S. M., \& Jorm, A. F. (2014). Parental factors associated with depression and anxiety in young people: A systematic review and meta-analysis. Journal of Affective Disorders, 156, 8-23. Doi: 10.1016/j.jad.2013.11.007.

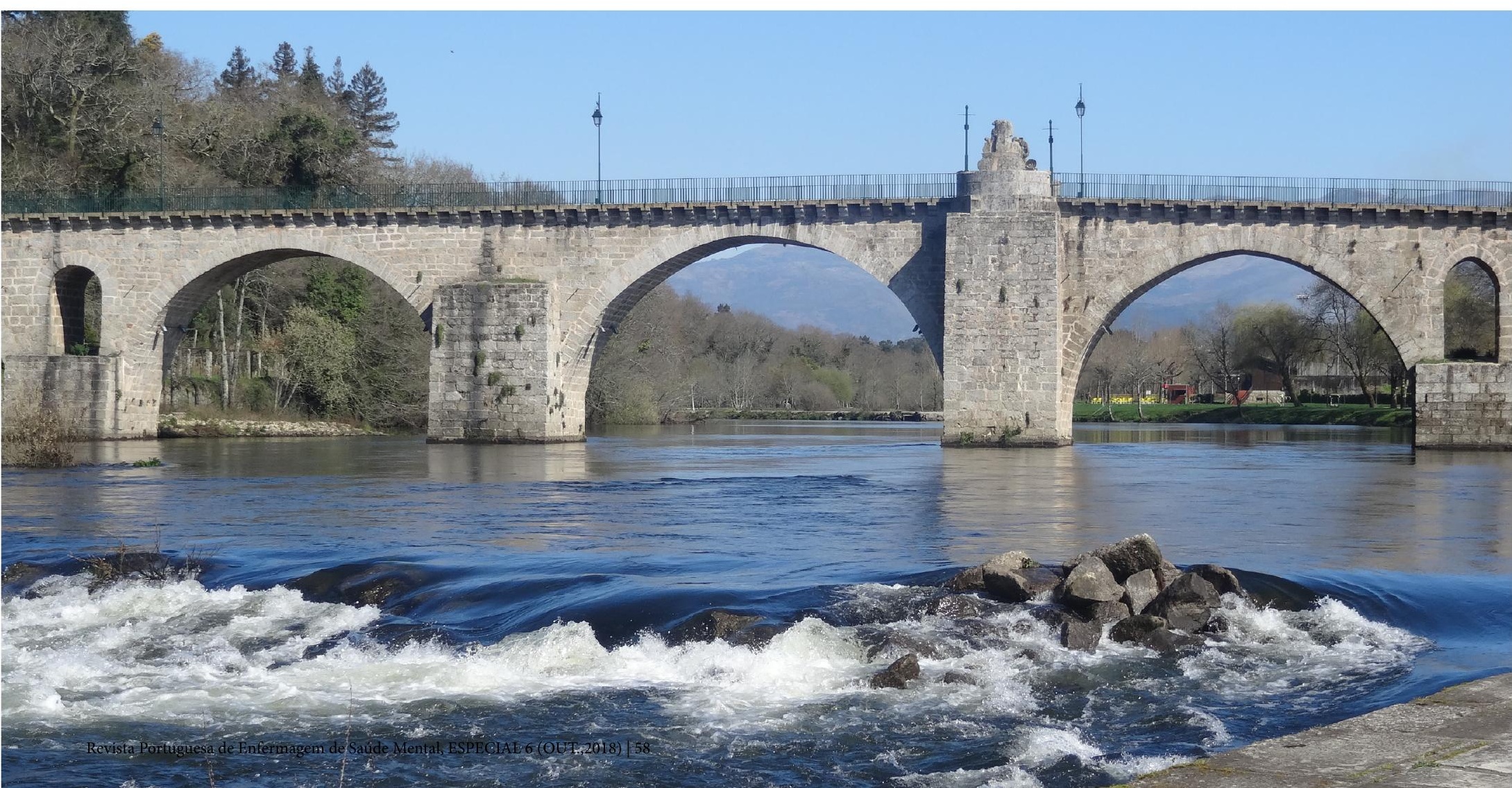

